\title{
THE HIDDEN H I-MASSIVE LUMINOUS INFRARED GALAXY HIZOA J0836-43: INSIDE-OUT GALAXY FORMATION ${ }^{1}$
}

\author{
M. E. Cluver,${ }^{2,5}$ T. H. Jarrett ${ }^{3}$ P. N. Appleton, ${ }^{4}$ R. C. Kraan-Korteweg, ${ }^{2}$ P. A. Woudt, ${ }^{2}$ B. S. Koribalski, ${ }^{6}$ \\ J. L. Donley, ${ }^{7}$ K. WAKamatsu, ${ }^{8}$ And T. Nagayama ${ }^{9}$ \\ Received 2008 June 6; accepted 2008 August 25; published 2008 September 23
}

\begin{abstract}
HIZOA J0836-43 is an extreme gas-rich $\left(M_{\mathrm{H}_{\mathrm{I}}}=7.5 \times 10^{10} M_{\odot}\right)$ disk galaxy which lies hidden behind the strongly obscuring Vela region of the Milky Way. Utilizing observations from the Spitzer Space Telescope, we have found it to be a luminous infrared starburst galaxy with a star formation rate of $\sim 21 M_{\odot} \mathrm{yr}^{-1}$, arising from exceptionally strong molecular PAH emission $\left(L_{7.7 \mu \mathrm{m}}=1.50 \times 10^{9} L_{\odot}\right)$ and far-infrared emission from cold dust. The galaxy exhibits a weak mid-infrared continuum compared to other star-forming galaxies and U/LIRGs. This relative lack of emission from small grains suggests atypical interstellar medium conditions compared to other starbursts. We do not detect significant [Ne v] or [O IV], which implies an absent or very weak AGN. The galaxy possesses a prominent bulge of evolved stars and a stellar mass of $4.4( \pm 1.4) \times 10^{10} M_{\odot}$. With its plentiful gas supply and current star formation rate, a doubling of stellar mass would occur on a timescale of 2 Gyr. Compared to local galaxies, HIZOA J0836-43 appears to be a "scaled up" spiral undergoing insideout formation, possibly resembling stellar disk building processes at intermediate redshifts.
\end{abstract}

Subject headings: galaxies: individual (HIZOA J0836-43) — galaxies: starburst — infrared: galaxies

\section{INTRODUCTION}

Understanding the fundamental origin and formation of galaxies requires the synthesis of multiwavelength observations and cosmological-based numerical simulations. Advances in both areas have created a compelling view of a cold dark matter dominated universe where structure forms hierarchically (White \& Rees 1978). Of fundamental importance to this formalism is insight into the formation and evolution of galaxy disks (Stringer \& Benson 2007).

Massive, gas-rich disk galaxies $\left(M_{\mathrm{H}_{\mathrm{I}}}>10^{10} M_{\odot}\right)$ are considered indicative of relatively unadvanced star building systems, making them ideal laboratories for testing this formalism. However, such systems are rare in the local universe and nearly all massive $\mathrm{H}$ I-rich disk galaxies are inactive or only passively forming stars (Sprayberry et al. 1995), thus providing few clues as to their formation and evolution. For example, Malin 1, an extreme case of a giant low surface brightness galaxy (Impey \& Bothun 1989), has a dormant star formation rate of $\sim$ $0.38 M_{\odot} \mathrm{yr}^{-1}$ (Rahman et al. 2007). In this Letter, however, we present evidence of an $\mathrm{H}$ I-massive disk galaxy, HIZOA J0836-43, undergoing a vigorous starburst.

This galaxy, discovered as part of a blind $\mathrm{H}$ I survey of the southern zone of avoidance, contains $7.5 \times 10^{10} M_{\odot}$ of $\mathrm{H} \mathrm{I}$ gas, has a total dynamical mass of $1.4 \times 10^{12} M_{\odot}$, and a 20 $\mathrm{cm}$ derived star formation rate of $\sim 35 M_{\odot} \mathrm{yr}^{-1}$ (Donley et al.

\footnotetext{
${ }^{1}$ This work is based on observations made with the Spitzer Space Telescope, which is operated by JPL/Caltech under a contract with NASA.

${ }^{2}$ Department of Astronomy, University of Cape Town, Rondebosch 7700, South Africa.

${ }^{3}$ IPAC, California Institute of Technology, Pasadena, CA 91125.

${ }^{4}$ NASA Herschel Science Center, California Institute of Technology, Pasadena, CA 91125

${ }^{5}$ IPAC Visiting Graduate Fellow, California Institute of Technology, Pasadena, CA 91125.

${ }^{6}$ Australia Telescope National Facility, CSIRO, Epping, NSW 1710, Australia.

${ }^{7}$ Steward Observatory, University of Arizona, Tucson, AZ 85721.

${ }^{8}$ Faculty of Engineering, Gifu University, Gifu 501-1193, Japan.

${ }^{9}$ Department of Astrophysics, Nagoya University, Chikusa-ku, Nagoya 4648602, Japan.
}

2006). It also appears to have a prominent bulge in the nearinfrared-bulge-to-disk ratio of $\sim 0.80$ in the $K_{s}$ band-central to an enormous, rapidly rotating $\mathrm{H}$ I disk.

The galaxy is located at $l=262.48^{\circ}, b=-1.64^{\circ}$, lying behind the Vela supernova remnant of the Milky Way. At optical wavelengths it is largely hidden by foreground gas and dust. We have therefore conducted a detailed infrared study of HIZOA J0836-43, using imaging and spectroscopy from the Spitzer Space Telescope, to fully reveal its morphology and past and present evolutionary state. Here we highlight key results from this study which provide new insights for understanding massive galaxy formation and evolution. The adopted distance of HIZOA J0836-43, $D_{L}=148 \mathrm{Mpc}$, is from Donley et al. (2006) as derived from its recessional velocity, $v_{\text {hel }}=$ $10689 \mathrm{~km} \mathrm{~s}^{-1}$.

\section{OBSERVATIONS AND DATA REDUCTION}

Near-infrared (NIR) and mid-infrared (MIR) imaging and spectroscopy served as primary data sets to study HIZOA J0836-43. From the ground, simultaneous $J H K_{s}$ images were obtained in 2006 April using the $1.4 \mathrm{~m}$ InfraRed Survey Facility (IRSF) and the 0.45" pixel scale SIRIUS camera (Nagayama et al. 2003), achieving an angular resolution of $\sim 1^{\prime \prime}$. The Spitzer Space Telescope was used to obtain imaging (Fazio et al. 2004; Rieke et al. 2004) and spectroscopy (Houck et al. 2004) in 2007 April and May. IRAC (3.6-8 $\mu \mathrm{m})$ achieves a spatial resolution of $\sim 2 "$ for all bands, and MIPS $(24,70,160 \mu \mathrm{m}) \sim 6^{\prime \prime}$, $18^{\prime \prime}$, and $40^{\prime \prime}$ for the $24,70,160 \mu \mathrm{m}$, respectively. Primary data reductions were done by the Spitzer Science Center (SSC) science pipeline (ver. S16.1.0) and using the SSC-developed MOPEX tool to produce final science-grade images. Galaxy photometry was performed using a matched elliptical aperture. The aperture was determined using the IRAC $3.6 \mu \mathrm{m}$ image, the optimal window to determine the shape and size of the galaxy after factoring in sensitivity, angular resolution, and foreground extinction. A symmetric isophotal fit of the light distribution down to the $1 \sigma$ sky level of this band was performed; the resulting aperture has a semimajor axis radius of $39.4^{\prime \prime}$, axis ratio of 0.42 , and a position angle of $\sim 110^{\circ}$. Foreground con- 

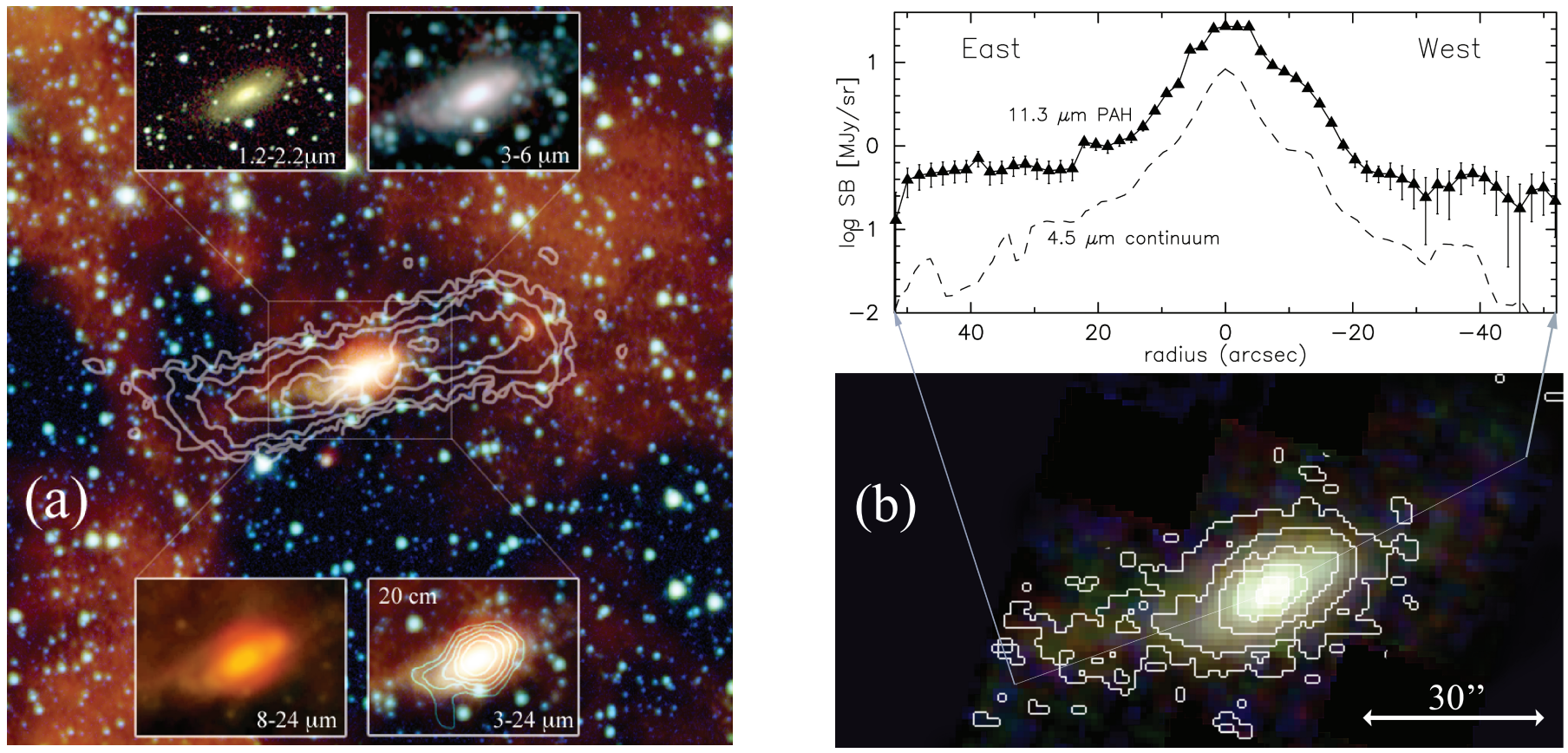

FIG. 1.-Infrared view of HIZOA J0836-43 through the Vela region of the Milky Way. (a) Composite 1-24 $\mu \mathrm{m}$ image of HIZOA J0836-43 ( 4' FOV = $\sim 170 \mathrm{kpc}$ ) with $\mathrm{H}$ I contours overlaid; insets show zoomed versions of the inner 1' region; $20 \mathrm{~cm}$ radio contours overlie the 3-24 $\mu \mathrm{m}$ inset. (b) Composite of spectral maps for the $6.6 \mu \mathrm{m}$ (blue), $7.7 \mu \mathrm{m}$ (green), and $11.3 \mu \mathrm{m}$ (red) PAH emission bands, overlaid with [Ne II] $12.8 \mu \mathrm{m}$ contours; the plot shows the 11.3 $\mu \mathrm{m}$ PAH and IRAC $4.5 \mu \mathrm{m}$ surface brightness across the east-west major axis, demonstrating the east warp and disk extended PAH emission relative to the bulgedominated stellar light.

taminating stars were masked from all images and replaced by the corresponding isophotal value of the source. The local background was determined from the median pixel value distribution within a surrounding annulus. The formal photometric uncertainties are $\sim 5 \%$ and $\sim 10 \%-20 \%$ for the NIR/IRAC and MIPS calibration errors, respectively.

IRS spectroscopy was obtained using the Short-Low (SL; 5-14 $\mu \mathrm{m}$ ), Short-High (SH; 10-20 $\mu \mathrm{m})$, and Long-High (LH; $19-38 \mu \mathrm{m})$ modules. Integration times of $3 \times 60 \mathrm{~s}$ were used for SL mapping $(R \sim 64-128)$, and $4 \times 30 \mathrm{~s}$ and $4 \times 14 \mathrm{~s}$ for SH and $\mathrm{LH}(R \sim 600)$ in staring mode. The data were first processed through the SSC S16.1.0 pipeline. The SL observations consisted of three separate mappings: center, east, and, west of the galaxy nucleus, each covering $0.4^{\prime} \times 0.7^{\prime}$ with $<10 \%$ overlap between adjacent maps. SH/LH observations were centered on the nucleus, and for background subtraction, a region $\sim 1^{\prime}$ south of the galaxy, not confused by foreground Galactic emission, was used. Spectral cubes and corresponding spectra were extracted using CUBISM (Smith et al. 2007). A 9.25" aperture was used for the nucleus (SL, SH, LH) and east and west disks (SL), and a 37" aperture for the entire galaxy (SL).

\section{RESULTS}

\subsection{Imaging and Photometry}

Figure $1 a$ shows a composite 1-24 $\mu \mathrm{m}$ image of the galaxy and its local environment $\left(\sim 4^{\prime}\right)$. The $\mathrm{H}$ I observations (Donley et al. 2006), also shown, demonstrate the enormous diameter $\left(\sim 3^{\prime}=\sim 130 \mathrm{kpc}\right)$ and $\mathrm{H}$ I mass $\left(M_{\mathrm{HI}}=7.5 \times 10^{10} M_{\odot}\right)$. The 1-5 $\mu \mathrm{m}$ window traces the evolved stellar population, while the MIR is sensitive to the interstellar medium: thermal dust continuum and emission from PAH (polycyclic aromatic hydrocarbon) molecules. PAHs produce broad emission bands in the MIR and are linked to ongoing or recent star formation (Allamandola et al. 1985). The emission likely arises from photodissociation regions (PDRs) which form adjacent to $\mathrm{H}$ II regions produced by star formation (Hollenbach \& Tielens 1997). The $20 \mathrm{~cm}$ radio continuum (Fig. 1a) is closely correlated with the 8-24 $\mu \mathrm{m}$ emission, indicating a common star formation origin. The infrared emission is clearly extended and exhibits asymmetry, or warp, along the eastern side in the PAH spectral map and surface brightness distribution along the major axis (Fig. 1b).

This extended morphology, also evident in the radio continuum, is reminiscent of a tidal tail, possibly due to a minor disturbance. At the Galactic location of HIZOA J0836-43 ( $l$ $=262.48^{\circ}, b=-1.64^{\circ}$ ) there is severe foreground dust obscuration. In order to estimate the Galactic extinction, we exploit the morphology-independent NIR colors of galaxies (Jarrett 2000), combined with the NIR sensitivity to the relatively well-modeled stellar population, by comparing the galaxy spectral energy distribution (SED) to dust-reddened templates for Population II-dominated galaxies (GRASIL code; Silva et al. 1998). The best-fit SED corresponds to a well-constrained extinction of $A_{V}=7.3 \pm 0.2 \mathrm{mag}$ using the Cardelli et al. (1989) extinction law convolved with the IRSF and IRAC bandpass filters. Table 1 presents the extinction-corrected global photometry and central surface brightness of the galaxy. The resulting SED is presented in Figure $2 a$. We include GRASIL templates for E, S0, and Sc types for comparison, as well as the spectrum of M82 (Sturm et al. 2000), the prototypical local starburst galaxy. The SED shows that in the MIR the galaxy resembles a Sc-type galaxy with strong emission from a dust continuum and PAH molecules. We see strong FIR emission longward of $60 \mu \mathrm{m}$ (see Table 1), indicating a significant cold dust component that dominates the total IR luminosity, $L_{\mathrm{TIR}}=$ $1.2 \times 10^{11} L_{\odot}$, giving rise to a luminous infrared galaxy 
TABLE 1

INFRARED PHOTOMETRY OF HIZOA J0836-43

\begin{tabular}{lcccccc}
\hline \hline Band & $\begin{array}{c}\lambda \\
(\mu \mathrm{m})\end{array}$ & $\begin{array}{c}A_{\lambda} \\
(\mathrm{mag})\end{array}$ & $\begin{array}{c}F_{\nu} \\
(\mathrm{mJy})\end{array}$ & $\begin{array}{c}\nu L_{\nu}{ }^{\mathrm{a}} \\
\left(10^{9} L_{\odot}\right)\end{array}$ & $\begin{array}{c}r_{\text {eff }} \\
(\operatorname{arcsec})\end{array}$ & $\mathrm{SB}\left(r_{\text {eff }}\right)^{\mathrm{b}}$ \\
\hline$I^{\mathrm{a}}$ & 0.82 & 4.2 & 28.12 & 69.2 & $\ldots$ & $\ldots$ \\
$J$ & 1.25 & 2.0 & 53.18 & 85.6 & 8.49 & 16.75 \\
$H$ & 1.63 & 1.3 & 60.53 & 73.1 & 7.88 & 16.03 \\
$K_{s}$ & 2.14 & 0.8 & 52.17 & 49.0 & 8.00 & 15.74 \\
IRAC-1 & 3.53 & 0.4 & 31.40 & 17.6 & 8.94 & 15.56 \\
IRAC-2 & 4.46 & 0.3 & 21.72 & 9.74 & 8.14 & 15.33 \\
IRAC-3 & 5.67 & 0.3 & 45.20 & 15.7 & 7.34 & 13.70 \\
IRAC-4 & 7.70 & 0.3 & 145.31 & 36.7 & 7.34 & 11.74 \\
MIPS-1 & 23.7 & $\ldots$ & 126.52 & 10.6 & $\ldots$ & $\ldots$ \\
MIPS-2 & 71 & $\ldots$ & 2291.3 & 65.9 & $\ldots$ & $\ldots$ \\
MIPS-3 & 156 & $\ldots$ & 3082.1 & 38.7 & $\ldots$ & $\ldots$ \\
Total IR & $3-1100$ & & & 119.5 & & \\
\hline
\end{tabular}

NoTES.-Aperture parameters: $a=39.35^{\prime \prime}, b / a=0.42, \phi=-70^{\circ}$. All measurements have been corrected for foreground dust and internal extinction; $A_{V}=7.3$. IRAC and MIPS measurements have been aperture corrected as follows: $0.940,0.974,0.871,0.814,1.107,1.240,1.705$ for 3.6, 4.5, 5.8, 8.0, $24,70,160 \mu \mathrm{m}$, respectively.

${ }^{\text {a }} D_{L}=148 \mathrm{Mpc}$ from Donley et al. (2006).

${ }^{\mathrm{b}}$ In units of mag $\operatorname{arcsec}^{-2}$.

${ }^{\mathrm{c}}$ Using the relation of Dale \& Helou (2002).

(LIRG). Fitting a two-component modified blackbody curve $(\beta$ $=1.5$ ) to this FIR continuum, we estimate a cold dust temperature of $\sim 30 \mathrm{~K}$. Assuming negligible contribution from the AGN, we estimate a total SFR of $\sim 21 M_{\odot} \mathrm{yr}^{-1}$ based on our global MIR measurements and the relation of Kennicutt (1998). This value is consistent with that of local LIRGS (Wang et al. 2006), but much higher than that of typical disk spirals (Kewley et al. 2002).

\subsection{MIR Spectroscopy}

The MIR spectroscopy of HIZOA J0836-43 is dominated by strong $\mathrm{PAH}$ emission that peaks in the nuclear region and appears extended compared to the distribution of stellar light, as traced by the $4.5 \mu \mathrm{m}$ emission (Fig. 1b). The nuclear light is shown in Figure $2 b$, which combines the SL, SH, and $\mathrm{LH}$ spectra, 5-36 $\mu \mathrm{m}$. The 6.2 and $7.7 \mu \mathrm{m}$ PAH features have equivalent widths of 0.72 and $0.82 \mu \mathrm{m}$, respectively. This is $\sim 20 \%$ and $50 \%$, respectively, larger than the average value $(0.53 \mu \mathrm{m})$ found by Brandl et al. (2006) for starburst galaxies. We attribute this to a combination of power in the PAH emission lines and the absence of a sharply rising dust continuum typical of starburst systems (e.g., M82 in Fig. 2a). The galaxy lies on the relation between $L(6.2 \mu \mathrm{m})$ and $\nu L_{\nu}(5.5 \mu \mathrm{m})$ found for starburst galaxies (see Fig. 8 of Desai et al. 2007), but at the highluminosity end. However, the flux density continuum ratio $f_{\nu}(6 \mu \mathrm{m}) / f_{\nu}(15 \mu \mathrm{m})$ of $\sim 1$ is indicative of a quiescent disk galaxy (Dale et al. 2000). Similarly, the rotational lines of $\mathrm{H}_{2}$, a direct tracer of the warm molecular component, are relatively weak in the galaxy. Using the relative excitations of the $S(1), S(2)$, and $S(3)$ lines, we estimate a warm molecular hydrogen temperature of $T \sim 330 \mathrm{~K}$ and a mass of only $\sim 1.3 \times 10^{7} \mathrm{M}_{\odot}$. Yet, the fine-structure lines of $\mathrm{Ne}, \mathrm{S}, \mathrm{Si}$, and Ar are all relatively strong; e.g., the [Ne III] 15.56/[Ne II] 12.81 ratio, which provides an indication of the hardness of the radiation field, is $\sim 0.11$, comparable to typical values for starbursting systems (Brandl et al. 2006). We do not detect significant [Ne v] or [O IV] which implies an absent or very weak AGN. Spectroscopy results are discussed in more detail in M. E. Cluver et al. (2008, in preparation).

\section{DISCUSSION}

For a galaxy in the local universe HIZOA J0836-43 possesses a number of unusual infrared properties; considered in combination with the massive reservoir of gas that feeds it, this could be a rare instance of a local galaxy undergoing insideout evolution, possibly resembling galaxy formation at earlier epochs. Here we compare properties of HIZOA J0836-43 with those of local and intermediate redshift samples.

The paucity of warm dust is evident from the weakly rising continuum seen in its spectrum (Fig. $2 b$ ), consistent with its low $L_{24 \mu \mathrm{m}} / L_{70 \mu \mathrm{m}}$ color compared to normal and star-forming systems, e.g., as compared to both the SINGS (Spitzer Infrared Nearby Galaxy Survey) sample, and the relatively nearby Great Observatories All-sky LIRG Survey (GOALS). In contrast, the strength of the PAH emission in HIZOA J0836-43, both in luminosity and relative strength of the bands compared to the continuum, is among the largest observed in any star-forming galaxy (Peeters et al. 2004), implying unusually strong PDR

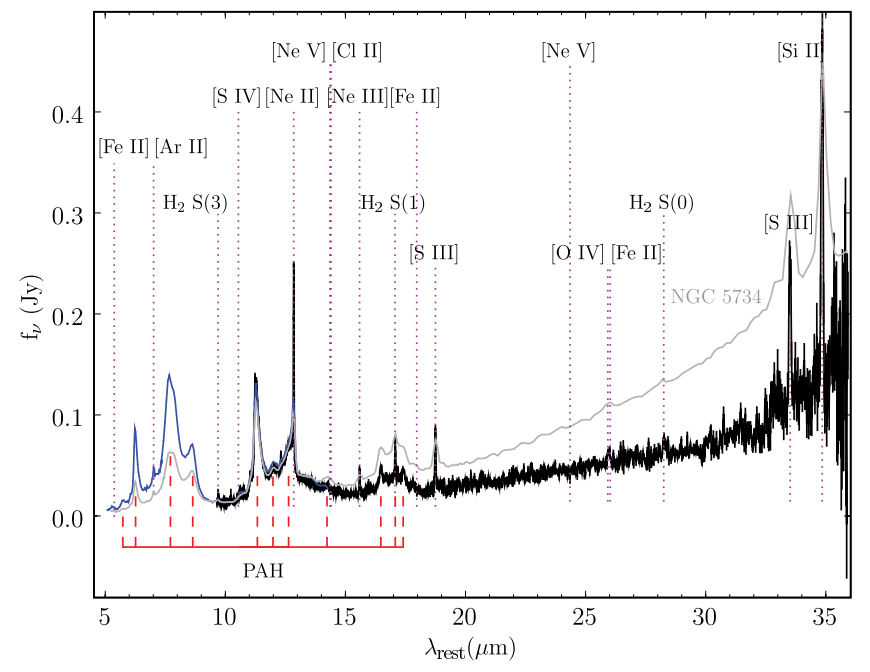

FIG. 2.- (a) Global SED with model templates and M82 spectrum for comparison. The templates are normalized to the galaxy $K_{s}$-band value. $(b)$ Combined low-resolution (blue) and high-resolution (black) spectrum for the massive galaxy nuclear region. For comparison, we show the SL/LL spectrum of the similarly cold GOALS galaxy NGC 5734 (gray). 
emission. These unusual MIR properties may arise from (1) a paucity of very small grains (VSGs) and/or (2) a soft UV radiation field. Powered by massive star formation, transiently heated VSGs are thought to be the source of MIR radiation. A weaker radiation field would give rise to cooler VSGs (Draine et al. 2007), which would reradiate at longer FIR wavelengths along with the large grain, cool $(T \sim 20 \mathrm{~K})$ component. Both mechanisms would be consistent with an evolutionary phase in which the activity is confined to heavily gas/dustobscured star forming regions, thus shielding the hard UV radiation from the rest of the disk, which in turn would yet to have experienced significant grain processing.

The growth progress of HIZOA J0836-43 is deduced from the stellar bulge population and current star formation rate. We estimate a stellar mass of $4.4( \pm 1.4) \times 10^{10} M_{\odot}$ for the galaxy (from the relation of Bell et al. 2003) and hence a specific star formation rate, SFR per stellar mass, of $\sim 0.5 \mathrm{Gyr}^{-1}$. Compared to local LIRGS (see Fig. 5 of Wang et al. 2006), this implies active stellar building as facilitated by its plentiful supply of gas, with a doubling of stellar mass in $\sim 2$ Gyr. The specific star formation rate of the galaxy in combination with its stellar mass appears typical for star-forming galaxies at $z \sim 0.7$, where gas fractions of disks were likely higher compared to local galaxies (Bell et al. 2005; Pérez-González et al. 2005). Figure 3 shows that the MIR luminosity is strongly correlated with $\mathrm{H}$ I content; even with its extreme H I mass, HIZOA J0836-43 appears consistent with being a "scaled up" disk galaxy, unlike Malin 1, which is explicitly quiescent by comparison. This suggests relatively "normal" evolution in HIZOA J0836-43, despite lying at the extreme high end (i.e., early evolutionary stage) of the relation.

HIZOA J0836-43 is a gas-rich spiral galaxy exhibiting a warp in its disk (Fig. 1a), likely the result of a disturbance in its recent past $(\ll 1 \mathrm{Gyr})$. Such an event could cause the observed starburst as gas from the extended $\mathrm{H}$ I disk flows into the central region of the galaxy. Hence the starburst is powered by gas consumption, as opposed to a major merger event. Gasrich galaxies, like HIZOA J0836-43, were likely more common in the distant universe and there is evidence that gas consumption and not merger interactions were driving stellar mass growth in the distant universe (Daddi et al. 2008). Recent work has suggested that many LIRGs seen at intermediate redshifts $(z \sim 0.8)$ achieve heightened star formation as a result of the high gas fractions of their disks and were less dependent on major interactions, compared to local LIRGs, to induce starburst activity (Melbourne et al. 2008; Marcillac et al. 2006).

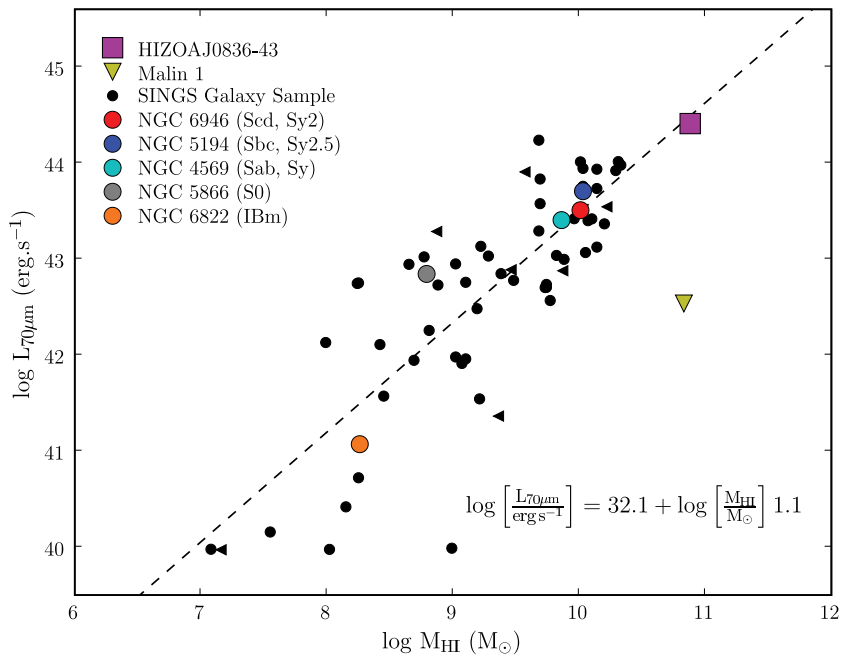

FIG. 3.-Infrared luminosity vs. H I mass, comparing HIZOA J0836-43, Malin 1, and the SINGS galaxy sample. For Malin 1 (yellow triangle) a 70 $\mu \mathrm{m}$ upper limit (Rahman et al. 2007) is shown; upper limits for H I mass are shown as black triangles.

Observational evidence suggests that disk galaxies evolve along the stellar mass-radius relation and have built stellar mass intensely since $z=1$, on average by means of inside-out growth (Barden et al. 2005; Trujillo et al. 2006). Comparing the extended regions of active star formation with the more centrally concentrated stellar bulge distribution (e.g., Fig. $1 b$ radial profile) suggests that HIZOA J0836-43 is undergoing vigorous disk building, an instance of inside-out growth. This combined with its PDR-dominated emission manifested as strong PAH emission coupled with a weak MIR continuum, makes it enigmatic in the local universe. Observing a galaxy at such a key point in its evolution could have far reaching implications for theories of galaxy formation and evolution.

We thank D. Dale and SINGS, J. Howell and GOALS for data access. We are grateful to S. Carey, G. Helou, S. Lord, J. Mazzarella, and B. Madore for insightful discussions. Support for this work was provided by NASA through an award issued by JPL/Caltech. M. C., R. K. K., and P. A. W. thank the NRF for financial support. M. C. thanks IPAC/Caltech for financial support through a Visiting Graduate Fellowship.

\section{REFERENCES}

Allamandola, L. J., Tielens, A. G. G. M., \& Barker, J. R. 1985, ApJ, 290, L25 Barden, M., et al. 2005, ApJ, 635, 959

Bell, E. F., et al. 2003, ApJS, 149, 289 2005, ApJ, 625, 23

Brandl, B. R., et al. 2006, ApJ, 653, 1129

Cardelli, J. A., Clayton, G. C., \& Mathis, J. M. 1989, ApJ, 345, 245

Daddi, E., et al. 2008, ApJ, 673, L21

Dale, D. A., \& Helou, G. 2002, ApJ, 576, 159

Dale, D. A., et al. 2000, AJ, 120, 583

Desai, V., et al. 2007, ApJ, 669, 810

Donley, J. L., et al. 2006, MNRAS, 369, 1741

Draine, B. T., et al. 2007, ApJ, 663, 866

Fazio, G., et al. 2004, ApJS, 154, 10

Hollenbach, D. J., \& Tielens, A. G. G. M. 1997, ARA\&A, 35, 179

Houck, J. R., et al. 2004, ApJS, 154, 18

Impey, C., \& Bothun, G. 1989, ApJ, 341, 89

Jarrett, T. H. 2000, PASP, 112, 1008

Kennicutt, R. C., Jr. 1998, ApJ, 498, 541
Kewley, L. J., et al. 2002, AJ, 124, 3135

Marcillac, D., et al. 2006, A\&A, 451, 57

Melbourne, J., et al. 2008, AJ, 135, 1207

Nagayama, T., et al. 2003, Proc. SPIE, 4841, 459

Peeters, E., Spoon, H., \& Tielens, A. 2004, ApJ, 613, 986

Pérez-González, P. G., et al. 2005, ApJ, 630, 82

Rahman, N., Howell, J. H., Helou, G., Mazzarella, J. M., \& Buckalew, B. 2007, ApJ, 663, 908

Rieke, G., et al. 2004, ApJS, 154, 204

Silva, L., et al. 1998, ApJ, 509, 103

Smith, J. D. T., et al. 2007, PASP, 119, 1133

Sprayberry, D., Impey, C. D., Bothun, G. D., \& Irwin, M. J. 1995, AJ, 109, 558

Stringer, M. J., \& Benson, A. J. 2007, MNRAS, 382, 641

Sturm, E., et al. 2000, A\&A, 358, 481

Trujillo, I., et al. 2006, MNRAS, 373, L36

Wang, J. L., et al. 2006, ApJ, 649, 722

White, S. D. M., \& Rees, M. J. 1978, MNRAS, 183, 341 